REVIEW

\title{
Treatment of severe psoriasis with infliximab
}

\author{
JA Leman \\ AD Burden \\ Alan Lyell Centre for Dermatology, \\ Western Infirmary, Glasgow, UK
}

\begin{abstract}
Psoriasis is a chronic, immune mediated inflammatory disease characterized by increased cell signalling via cytokines and chemokines on a background of up-regulated gene expression. There is substantial evidence that psoriasis should be regarded as more than a cutaneous disease; major psychological morbidity and increased mortality from cardiovascular disease and cancer are increasingly recognized. Improved understanding of the genetic and immunological mechanisms underpinning psoriasis has occurred concurrently with the development of targeted biological therapies including infliximab. These newer therapeutic approaches can be very effective but their long term safety profile is not yet fully determined.
\end{abstract}

Keywords: psoriasis, pathogenesis, co-morbidities, treatment, infliximab

\section{Introduction and clinical features}

Psoriasis, a chronic inflammatory disease, affects $1.5 \%$ to $2.5 \%$ of the UK population and is diagnosed on clinical grounds (Gelfland et al 2005). A relapsing and remitting course is characteristic. There are several different phenotypes which frequently overlap and switching between phenotypic groups may occur. Around $80 \%$ of individuals present with chronic plaque psoriasis characterized by well demarcated, red, thickened patches of skin which become elevated and covered with an adherent, silvery scale (Lebwohl and Callen 2003). Typically plaques are located on the extensor aspect of knees, elbows, hairline, scalp, intergluteal cleft and lumbosacral area. Nail involvement, which occurs in $30 \%$ to $50 \%$ of patients, is characterized by pitting of the nail surface, onycholysis (separation of nail plate from the nail bed) and subungal hyperkeratosis (Scher 1985). Guttate psoriasis is typified by the acute onset of multiple small patches predominantly located on the trunk. This form may be the initial presentation in children with a family history of psoriasis. In up to $80 \%$ of cases there is a history of a preceding sore throat (Telfer et al 1992). Erythrodermic psoriasis denotes the involvement of the entire skin surface which, although rare, is significant because it can be life threatening with a risk of sepsis and thermoregulatory disruption. Pustular psoriasis may be localized or generalized. In the generalized variant sheets of sterile pustules are found on a background of red skin. Fever, arthralgia and malaise are common accompaniments. In palmoplantar pustulosis clusters of sterile pustules stud the thenar and hypothenar eminences as well as the plantar surface, especially the instep. Controversy exists as to whether or not these pustular eruptions are part of the psoriasis spectrum or are separate entities. Psoriatic arthritis affects between $5 \%$ and $30 \%$ of psoriasis patients and can be aggressive and deforming. Both the axial and the peripheral joints may be involved with several overlapping patterns: asymmetric, oligoarticular disease, predominant distal arthritis characterized by involvement of the distal interphalangeal joints, arthritis mutilans, symmetric polyarthritis (closely resembling rheumatoid arthritis) and axial involvement/spondolarthropathy (Espinoza et al 1992; Moll et al 1973). Enthesopathy and dactylitis are characteristic features.

Symptoms of psoriasis commonly include itching and tightness of the skin as well as fatigue (Burd 2006). At diagnosis, patients are educated as to the likely requirement 
for life-long treatment. Spontaneous remission is reported but relapses are common. The importance of recognizing common co morbidities such as cardiovascular disease and metabolic syndrome is increasingly being recognized.

\section{Comorbidities and risk factors}

As is the case with other chronic inflammatory diseases, psoriasis is associated with increased cardiovascular morbidity. In patients with severe psoriasis, the overall cardiovascular mortality risk for patients admitted to hospital at least once for their skin, is increased by 50\% (SMR 1.52; 95\% CI; 1.44-1.60) (Mallbris et al 2004). Psoriasis was demonstrated to be an independent risk factor for myocardial infarction in a population based cohort study. The risk is greatest for younger patients with severe disease even when corrected for traditional vascular risk factors (Gelfand et al 2006).

The increased cardiovascular morbidity and mortality in patients with psoriasis is likely due to several interrelated factors including: smoking, alcohol consumption, dyslipidemia, obesity, chronic inflammation. The risk of psoriasis is higher in smokers and former smokers than in those who have never smoked (Lebowohl and Callen 2006). Alcohol consumption is increased in patients with psoriasis (Poikolainen et al 1999). Increased rates of hyperlipidemia occur in those with severe, long standing psoriasis (Uyanik et al 2002). The prevalence of obesity is twice that of the normal population (Lebowohl and Callen 2006). A recent study identified increased adiposity and weight gain as risk factors for incident psoriasis (Setty et al 2007).

As well as physical morbidity, the psychological and psychosocial effects of psoriasis are increasingly being recognized. The impact on quality of life equates to, and sometimes exceeds, that found in hypertension, heart disease, diabetes and cancer (Rapp et al 1999).

\section{Assessing disease severity}

When categorizing psoriasis as mild, moderate or severe, physicians may consider several parameters including: response to treatment, impact on quality of life (Dermatology Life Quality Index; DLQI), body surface area involved (Physician Global Assessment; PGA), disease activity (plaque redness, scale and thickness - psoriasis area and severity index).

Psoriasis Area and Severity Index (PASI) is considered by many to be the gold standard severity assessment tool and consists an average measure of 4 parameters; redness, thickness and scaliness of lesions and extent of disease.
Each parameter is graded as $0-4$ with 0 equating to no disease and 4 severe involvement. The body is divided into 4 areas (head, arms, trunk to groin, legs to top of buttocks). The scores are summed, a percentage of the area covered by psoriasis estimated and a multiplier applied to each area generating a final PASI. PASI ranges from 0 to 72 with PASI $>10$ recognized as indicative of severe disease (Feldman and Krueger 2005; Finlay 2005). A 75\% reduction in PASI from baseline (PASI 75) is commonly used as an indicator of disease response, and in most clinical trials this is the primary efficacy endpoint. For individual patients a smaller improvement in PASI can be clinically meaningful (Carlin et al 2004). This is reflected in clinical trials which frequently report PASI 50 rates as well as PASI 75.

In day to day practice, a PGA is commonly used. A static form records disease at a solitary time point. In contrast, the dynamic form assesses global improvement compared to baseline (Feldman et al 2005). A static PGA score ranges from 0 (no psoriasis) to 7 (severe disease).

Quality of life is most frequently assessed using a skinspecific 10 point questionnaire, the DLQI. This assesses the impact of disease over 6 domains each on a scale of 0 (not at all) to 3 (very much). A score of 0 indicates that psoriasis or its treatment is not impacting on health related quality of life at all. In contrast, a score $>10$ indicates a very large effect on life quality (Lewis et al 2004; Findlay 2005a). A change of 5 points is considered to be clinically relevant (Mease et al 2006).

\section{Economic impact of psoriasis}

Patients with severe disease represent around $20 \%$ to $30 \%$ of all psoriasis patients. Systemic therapies are frequently required and the economic burden for patients and health service alike is considerable. United Kingdom estimates of cost of treatment for moderate to severe disease vary from $£ 45.5$ to $£ 80$ million (Van de Kerkhof 2003) Costs for individual patients are considerable and may include difficulties with employment (NICE 2006; Scottish Medicines Consortium 2007).

\section{Epidemiology and genetics}

A quarter of patients develop the disease before the age of 20 years. A further peak in incidence is recorded in the fifth and sixth decades (Lebwohl 2003; Burd 2006). The age of onset seems to be genetically determined. Men and women are equally frequently affected. Caucasians are more commonly affected than other ethnic groups (Lebwohl 2003). Environmental factors may trigger or exacerbate existing disease. 
Streptococcal antigens have been the focus of much research. The streptococcal M protein is homologous to the $50 \mathrm{kDa}$ type I keratin, $\mathrm{K} 14$ and a $67 \mathrm{kDa}$ streptococcal protein is homologous to myosin and the beta chain of the class II HLA antigens. These observations suggest that molecular mimicry between streptococcal protein and keratin may, in some individuals, lead to activation of autoreactive $\mathrm{T}$ cells (Valdimarsson et al 1997; Gudmundsdottir et al 1999; Camp et al 2006).

Familial clustering and high concordance rates in monozygotic twins indicate that psoriasis is highly heritable (Lomholt 1963). Linkage analysis has revealed multiple susceptibility loci with evidence for a major risk gene on chromosome 6p (PSORS 1) highly associated with HLA-Cw6 (Elder 2006). Interestingly, several other suspected loci are shared with other inflammatory and autoimmune conditions such as inflammatory bowel disease and atopic dermatitis (Cookson et al 2001). A large number of possible susceptibility polymorphisms are being described in large scale whole genome association scans, including polymorphisms in the IL-23 receptor and its ligand (Capon et al 2007).

\section{Pathogenesis}

Microscopically, psoriasis is characterized by a thickened epidermis with the retention of nucleated keratinocytes in the stratum corneum, loss of keratinocyte differentiation, an inflammatory cell infiltrate, mainly neutrophils and lymphocytes and vascular proliferation. T cells have been shown to play a pivotal role in pathogenesis driving epidermal hyperplasia. Multiple studies have demonstrated the importance of specific T cell subsets and dendritic antigen presenting cells (Gilhar et al 1997; Krueger et al 2002). The cytokine network model proposes activation of immunocytes by, for example, infections, medications and trauma, triggering a cascade of cytokines, chemokines and growth factors (Nickoloff 1999). Tumor necrosis factor alpha (TNF alpha) produced by antigen presenting cells, T cells and keratinocytes, is known to be elevated in psoriatic plaques, and serum levels correlate with disease activity. New biological interventions have targeted $\mathrm{T}$ cells, antigen presenting cells and TNF alpha.

\section{Therapeutic options for psoriasis}

For most patients, topical therapies supervised by their primary care physicians, suffice. Topical corticosteroids, vitamin $\mathrm{D}$ analogs, coal tar and tazoretene are commonly prescribed (Bundu-Kamara 2002; Clements et al 2006; Smith et al 2006) For those with moderate-severe disease, usually defined as disease affecting $>5 \%$ to $10 \%$ of body surface area (BSA), topical treatment can be labor intensive and messy to apply. Systemic therapies and phototherapies are, for this group, often more practical and efficacious (Cather et al 2005).

In addition, more limited psoriasis affecting only the face, hands or feet, may merit systemic intervention because of cosmetic or functional difficulty. Phototherapy using either narrow band ultraviolet B or photochemotherapy using long wave ultraviolet $\mathrm{A}$ is appropriate for those with an inadequate response to topical treatments or for those with extensive disease (Bandu-Kamara 2002). Treatment is usually delivered 2 to 3 times a week in dedicated centers staffed by appropriately trained individuals. Skin burning, photoaging and skin cancer may be limiting factors. For those who fail to respond or those in whom relapse after treatment is rapid, systemic therapies such as methotrexate, acitretin, ciclosporin and fumaric acid esters, may be employed (Lebwohl 2003). These are prescribed by hospital specialists and require close supervision and monitoring. All patients are fully counselled regarding potential adverse effects and toxicity. Regular monitoring is essential. Even with the use of systemic therapies, additional topical applications are frequently used during disease exacerbations or at therapy resistant sites.

\section{Place of infliximab in treatment of psoriasis}

Currently available biological treatments in Europe comprise 3 TNF alpha antagonists (infliximab, adalimumab and etanercept) and the $\mathrm{T}$ cell targeted treatment, efalizumab. The licensed indication for each of these is similar: moderate to severe plaque psoriasis in patients who have failed to respond to, have a contraindication to or are intolerant of other systemic therapies including ciclosporin, methotrexate and psoralen and ultraviolet A light (PUVA). In the US, a second $\mathrm{T}$ cell targeted treatment (alefacept) is also available. The licensed indication for biological treatment is also different in the US, with no requirement for patients to have failed, or be intolerant of other systemic agents. Ustekimumab, a monoclonal antibody directed against IL-12 and IL-23, has completed phase 3 trials and is awaiting marketing authorization from the licensing agencies in Europe and the US.

\section{Infliximab}

Infliximab $\left(\right.$ Remicade $^{\circledR}$ ) is a chimeric (75\% human, $25 \%$ murine) IgG1 monoclonal antibody which targets TNF alpha. Infliximab binds with high affinity to both soluble and membrane-bound TNF alpha and inhibits the ability of 
TNF alpha to bind to the TNF receptor. Infliximab is licensed for use in rheumatoid arthritis, Crohn's disease, anklyosing spondylitis and psoriatic arthritis. For patients with psoriasis, infliximab is infused at a dose of $5 \mathrm{mg} / \mathrm{kg}$ at week 0,2 and 6 and then at 8- to 12-weekly intervals thereafter. There are data on efficacy in psoriasis from psoriatic arthritis trials and also from trials primarily designed to investigate efficacy in plaque psoriasis.

A double-blind, placebo-controlled phase 2 trial (SPIRIT) randomized 249 patients in a 2:2:1 ratio to receive infliximab $3 \mathrm{mg} / \mathrm{kg}, 5 \mathrm{mg} / \mathrm{kg}$ or placebo administered at weeks 0,2 and 6 . Randomized patients had plaque psoriasis of at least 6 months duration, and PASI $\geq 12$ and plaques covering $\geq 10 \%$ BSA. The median baseline PASI was 20, 20 and 18, median BSA $29 \%, 25 \%$ and $26 \%$ for $3 \mathrm{mg} / \mathrm{kg}, 5 \mathrm{mg} / \mathrm{kg}$ and placebo groups, respectively. All patients had systemic therapies or light therapy discontinued 1 month before and during the trial. The primary endpoint was the proportion of patients achieving a PASI 75 at week 10 . At week 26 patients with a PGA $\geq 3$ were eligible for a single further infusion. Patients were followed up to week 26 to assess duration of response. At week $10,72 \%$ of the $3 \mathrm{mg} / \mathrm{kg}$ and $88 \%$ of the $5 \mathrm{mg} / \mathrm{kg}$ group had achieved a PASI 75 . In contrast, only $6 \%$ of placebo group achieved the primary endpoint $(\mathrm{p}<0.001)$. PGA results corroborated PASI results with $72 \%$ and $90 \%$ of patients in the infliximab 3 and $5 \mathrm{mg} / \mathrm{kg}$ groups respectively achieving PGAs of minimal or cleared psoriasis at week 10. Median baseline DLQI scores were 11,12 and 14 for $3 \mathrm{mg} / \mathrm{kg}, 5 \mathrm{mg} / \mathrm{kg}$ and placebo respectively. The median changes in DLQI from baseline to week 10 were -8 and -10 for the 3 and $5 \mathrm{mg} / \mathrm{kg}$ groups respectively compared to no change in the placebo group ( $\mathrm{p}<0.001$ ) (Gottlieb et al 2004).

In a phase 3 , multi-center, double-blind placebo controlled trial at 32 centers in Europe and Canada (EXPRESS), 378 patients with plaque psoriasis were randomized in a 4:1 ratio to infliximab $5 \mathrm{mg} / \mathrm{kg}$ or placebo administered at week $0,2,6$ and then at 8 weekly intervals to week 46 (Reich et al 2005). At week 24 placebo-treated patients crossed over to infliximab. Those eligible for inclusion had moderate to severe plaque psoriasis with $\mathrm{PASI} \geq 12$ and a $\mathrm{BSA}>10 \%$ affected where PUVA or systemic therapies would have been appropriate. Systemic and photo therapies were stopped 1 month before entry and during the trial. The groups were comparable in terms of baseline PASI, body surface area involvement and nail severity index. The primary endpoint was the proportion of patients achieving a PASI 75 after 10 weeks. The secondary endpoints included the PASI 50 and PASI 90 at week 10. Intention to treat analysis was used for primary endpoints and non ITT analysis for secondary endpoints such as PASI 50, 75 and 90 at week 24 and 50. After 10 weeks $80 \%$ of patients achieved a PASI 75 and $57 \%$ a PASI 90 compared to $3 \%$ and $1 \%$ in the placebo group respectively ( $\mathrm{p}<0.0001)$. Response was maintained at week 24 with a PASI 75 of $82 \%$ and PASI 90 of $58 \%$ in infliximab group. By week 50 the proportion achieving a PASI 75 and PASI 90 had fallen to $61 \%$ and 45\% respectively. $83 \%$ of patients achieved a PGA of clear or minimal at week $10(\mathrm{p}<0.0001)$ but by week 50 this had fallen to $53 \%$. A response to treatment was seen as early as week 2 . By week 6, significantly more infliximab patients had achieved a PASI 75 and PASI 90 ( $\mathrm{p}<0.0001)$. The loss of clinical response correlated with low infliximab serum concentrations which was partly attributed to the development of anti-infliximab antibodies.

The suggestion that for some patients more regular infusions are required was investigated in a further study (EXPRESS II) which compared regular versus on-demand maintenance regimens (Menter et al 2007). In this randomized, double-blind placebo-controlled trial eligible patients were randomly assigned to placebo or infliximab in a 2:3 ratio. Induction doses of infliximab were either 3 or $5 \mathrm{mg} / \mathrm{kg}$ given at week 0,2 and 6 . At week 14, patients in the infliximab group were randomized again to either 8 weeks continuous maintenance therapy or as required therapy at the same dose administered in the induction phase. As required infliximab was given when the PASI improvement from baseline was less than $75 \%$ at the 4-weekly study visits. Patients in the placebo group were crossed over at week 16 to receive infliximab $5 \mathrm{mg} / \mathrm{kg}$ at week 16, 18 and 22 and every 8 weeks to week 46. All those enrolled had moderate to severe psoriasis (eligible for treatment with systemic therapies or phototherapy) with a PASI $\geq 12$ and plaques covering at least $10 \%$ BSA. At week $10,75.5 \%$ of the $5 \mathrm{mg} / \mathrm{kg}$ infliximab group and $70.3 \%$ of the $3 \mathrm{mg} / \mathrm{kg}$ group compared to $1.9 \%$ of the placebo group had achieved a PASI 75. PASI 90 (considered almost clear) was achieved by $45.2 \%$ of those treated with $5 \mathrm{mg} / \mathrm{kg}$ infliximab and $37.1 \%$ of $3 \mathrm{mg} / \mathrm{kg}$ infliximab compared to $0.5 \%$ of controls, $\mathrm{p}<0.001$. PASI responses were better maintained with continuous compared with intermittent therapy within each dose and with $5 \mathrm{mg} / \mathrm{kg}$ compared with $3 \mathrm{mg} / \mathrm{kg}$. PGA responders, at week 10 , were $76 \%$ in the $5 \mathrm{mg} / \mathrm{kg}$ infliximab group, $69.8 \%$ in $3 \mathrm{mg} / \mathrm{kg}$ group and $1 \%$ in the placebo arm. PGA responders were classified as $100 \%$ clear (clear) or $75 \%$ to $99 \%$ clear (excellent clearing) relative to baseline. 
Patients receiving infliximab had more frequent serious adverse events than placebo in all studies $6.1 \%$ vs $0 \%$ in SPIRIT, $6 \%$ (this included one fatal infection in the infliximab arm) vs 3\% EXPRESS and $2.9 \%$ in $5 \mathrm{mg} / \mathrm{kg}$ group and $1 \%$ in $3 \mathrm{mg} / \mathrm{kg}$ group versus $2.4 \%$ placebo in EXPRESS II. Laboratory abnormalities were uncommon, the most frequently observed being an elevation in liver enzymes. Infusion reactions occurred in $20 \%$ in SPIRIT compared to $2 \%$ placebo. It is not possible to ascertain the number of infusion reactions in EXPRESS as data are presented as percentage of total number of infusions. Infusion reactions were more common when the drug was given on an as required basis rather than as planned regular infusions. Twenty-three percent of patients in SPIRIT at week 26 and $22 \%$ at week 46 in EXPRESS developed antibodies. There were 2 cases of tuberculosis in the infliximab group in EXPRESS II. There is some concern that infliximab, in common with other biological therapies, may increase the risk of malignancies. Three patients in EXPRESS had skin cancers. The follow-up period in these trials is short and the long term safety data are not currently available nor can they be extrapolated from other specialities that may have longer experience in the use of this drug.

\section{Quality of life}

Trials consistently demonstrate an improvement in quality of life comparing baseline with week 10 assessments with infliximab compared to placebo (Gottlieb et al 2004; Reich et al 2005; Menter et al 2007). At week 50, however, the improvements in quality of life were smaller than at week 10 in two studies (Reich et al 2005; Menter et al 2007). Specific analysis of health related quality of life was undertaken by Feldmann et al as part of the SPIRT trial. Infliximab $3 \mathrm{mg} / \mathrm{kg}$ and $5 \mathrm{mg} / \mathrm{kg}$ groups showed a median percentage improvement in DLQI scores of $84 \%$ and $91 \%$ respectively compared to $0 \%$ in the placebo group $(\mathrm{p}<0.001)$. The median decrease from baseline DLQI score at week 10 was 8 and 10 for the 3 and $5 \mathrm{mg} / \mathrm{kg}$ groups respectively compared to 0 for placebo $(\mathrm{p}<0.001)$. Forty percent of the $5 \mathrm{mg} / \mathrm{kg}$ patients had a DLQI of 0 at week 10 compared to $33 \%$ in $3 \mathrm{mg} / \mathrm{kg}$ group and $2 \%$ in placebo group. There was a strong correlation between the percentage change in DLQI from baseline to week 10 and the PASI scores (Feldman et al 2005).

\section{Infliximab for psoriatic arthritis}

There have been two randomized controlled trials of infliximab in psoriatic arthritis. The outcome measures commonly used are the American College of Rheumatology (ACR) response criteria, the Psoriatic Arthritis Response Criteria (PsARC) and health assessment questionnaire (HAQ).

The ACR is a composite score which compares at baseline, and then at defined time points thereafter, the tender and swollen joint score and 3 out of 5 other measures: patient global assessment, physician global assessment, patient pain, heath assessment questionnaire and acute phase reactant (erythrocyte sedimentation rate or $\mathrm{C}$ reactive protein). An ACR 20 response would be constituted by a $20 \%$ improvement in tender and swollen joint count and a 20\% improvement of at least 3 of the 5 other elements in the composite criteria. ACR 50 and 70 represent, respectively, at least a 50\% and $70 \%$ improvement. Other studies have used PsARC which requires at least $30 \%$ improvement of tender or swollen joint count as well as a 1 point improvement on a 5-point scale in patient and or physician global assessment and no worsening of any score. Some investigators use a measure of physical function, the Health assessment Questionnaire (HAQ), which, in studies of rheumatoid arthritis, has been shown to be highly discriminative and to correlate well with response to therapy (Mease et al 2005).

In a randomized, double-blind, placebo-controlled trial in 104 patients with psoriatic arthritis (IMPACT) at 16 weeks $65 \%$ of patients treated with infliximab achieved an ACR 20 compared with $10 \%$ in the placebo arm. (RR 6.80, 95\% CI 2.89 to 16.01). Forty-six percent of the infliximabtreated patients achieved an ACR 50 (RR 49.00, 95\% CI 3.06-785.06) and 29\% achieved an ACR 70 (RR 31.00 95\% CI 1.90-504.86) compared with none in the placebo group. Seventy-five percent of infliximab-treated patients achieved a PsARC (RR 3.55, 95\% CI 2.05-6.13). Functional improvement was demonstrated by improvement in HAQ scores with infliximab compared to placebo - mean difference 51.4 (95\% CI 48.8-54.72). Radiographic assessments were not performed so the potential of infliximab to delay progression of joint disease could not be commented upon (Antoni et al 2005a).

In the phase 3, double-blind, placebo-controlled trial (IMPACT II) 200 patients with active psoriatic arthritis were treated with infliximab $5 \mathrm{mg} / \mathrm{kg}$ or placebo at weeks $0,2,6$ and every 8 weeks thereafter up to 1 year (Kavanaugh et al 2007). Concomitant methotrexate was permitted but was not mandatory. Those randomized to placebo crossed over to infliximab at week 24. Dose escalation to $10 \mathrm{mg} / \mathrm{kg}$ at week 38 , was permitted in those who had no response or had lost response. ACR 20, ACR 50 and ACR 70 responses were maintained with continued infliximab treatment in the randomized infliximab group and were substantial following crossover to infliximab 
in the placebo/infliximab group. For example, ACR 20 was achieved by $58.9 \%$ and $61.4 \%$ of patients in the randomized infliximab and placebo/infliximab groups, respectively. At week 54, the ACR response was consistent whether or not methotrexate had been used. For example, ACR 20 was achieved by $56.8 \%$ of patients on infliximab plus methotrexate and $60.9 \%$ of patients on infliximab alone. Other efficacy results such as PSARC and duration of morning stiffness were also maintained through to week 54 .

\section{Infliximab in nail psoriasis}

Efficacy of infliximab in nail psoriasis has been documented in case reports, case series and as part of large clinical trials of infliximab in plaque psoriasis. In a phase 3 study of 378 patients with moderate to severe psoriasis, $81.8 \%$ had nail psoriasis at baseline. Infliximab $5 \mathrm{mg} / \mathrm{kg}$ was given at week 0, 2 and 6 then 8 weekly to week 46. The Nail Psoriasis Severity Index (NAPSI) at baseline was 4.6 in treatment group and 4.3 in the placebo group. At week 10 and 24 the mean NAPSI scores had improved by $26.8 \%$ and $57.2 \%$ respectively and $-7.7 \%$ and $-4.1 \%$ in the placebo group (both $\mathrm{p}<0.001)$. Psoriasis in the target nail had completely cleared in $6.9 \%$ in patients at week 10 and $44.7 \%$ at week 50 versus $5.1 \%$ in placebo group ( $\mathrm{p}<0.001$ ) (Rich et al 2008).

A small study of 25 patients treated with infliximab $5 \mathrm{mg} / \mathrm{kg}$ at week $0,2,6,14$ and 22 resulted in a NAPSI 75 (clinical remission) in all patients by week 22. Remission was maintained at follow up 12 weeks after the final infusion (Bianchi et al 2005).

In a small, non-randomized, open-label study of 18 patients mean NAPSI declined from 55.8 at baseline to 29.8 at week 14. At week 38, after 6 infusions, there was almost complete resolution of nail involvement and improved quality of life (Rigopoulos et al 2008).

\section{Infliximab in special situations: psoriasis in children, erythrodermic and pustular psoriasis}

At present, there are no clinical trials of infliximab in childhood psoriasis. Case reports suggest efficacy in psoriasis, including pustular disease and psoriatic arthropathy (Menter et al 2004; Pereira et al 2006; Rott et al 2007).

One small series, which incorporates details of previous case reports, suggests that infliximab is a useful treatment in erythrodermic psoriasis (Takahashi et al 2007). The patients described frequently had life threatening disease highly resistant to multiple previous therapies. Response to infliximab, which may be combined with systemic therapies such as methotrexate or acitretin, is often prompt but long term follow-up data are scant.

There are several case reports and case series indicating efficacy of infliximab (used off label) in pustular psoriasis (Trent et al 2004; Schmick et al 2004; Lewis et al 2006; Weisenseel et al 2006; Vieira et al 2008). Infliximab is usually administered every 6 weeks at a dose of $5 \mathrm{mg} / \mathrm{kg}$ sometimes with the addition of weekly, low dose methotrexate (Routhouska et al 2008). At a molecular level, infliximab has been shown to down regulate disease-promoting chemokines such as interleukin 8, GRO-alpha and MCP-1 in pustular psoriasis (Benoit et al 2004). Most reports include only small numbers of patients with limited follow-up data.

Paradoxically, treatment with infliximab has been implicated in the development of pustular psoriasis (Martínez-Morán et al 2007) and palmoplantar pustulosis (Mossner et al 2008). In the latter report, 5 patients with chronic plaque psoriasis developed palmoplantar pustulosis during or after discontinuation of infliximab. A review of TNF alpha-induced psoriasis or psoriasiform eruptions was published in 2008. Sixty-three of the 120 affected patients had been treated with infliximab. Psoriasis, palmoplantar pustulosis and nail psoriasis had been induced immediately after one infusion through to 63 months post treatment. The underlying mechanism of induction of psoriasis/psoriasiform exanthema in these patients remains elusive (Wollina et al 2008). Patients who have developed psoriasis or a psoriasiform eruption during treatment with one TNF blocker may respond, without adverse event, to an alternative TNF antagonist (Collamer et al 2008).

\section{Infliximab and pregnancy}

TNF antagonists are listed as pregnancy category B drugs: animal studies have failed to demonstrate a risk to the fetus but there are no adequate and well controlled studies of the effect of the drugs on pregnant women. In a series of 32 women, identified from the British Society for Rheumatology Biologics Register, who were exposed to anti TNF drugs at the time of conception, or in the preceding 10 months, $91 \%$ elected to continue their pregnancies. Seventy-six percent delivered health babies and $24 \%$ had first trimester miscarriages, a rate consistent with that expected in the general population (Hyrich et al 2006).

\section{Safety of and adverse events associated with infliximab}

Infliximab is generally well tolerated. Regular infusions in a hospital setting provide an opportunity to monitor for potential adverse events. 
Most safety data come from the rheumatoid arthritis and Crohn's populations. Infliximab has been used extensively in the UK by rheumatologists who had the foresight to establish a biologics registry under the auspices of the British Society of Rheumatology. It is estimated that around $80 \%$ of patients commencing biological therapies are entered on the register, enabling comprehensive data collection on adverse events.

Absolute contraindications to commencement include active infections, recurrent or chronic infections, previous untreated tuberculosis (TB), moderate to severe cardiac failure and recent history of malignancy (except for skin cancer such as basal cell carcinoma). In clinical trials upper respiratory tract infection, sinusitis, nausea and headache are the most commonly reported adverse events (Scheinfield 2004; Hochberg et al 2005).

\section{Infections (including TB)}

The most important adverse event of infliximab is the increased risk of infection including sepsis and opportunistic infection. Patients with predisposing underlying conditions such as diabetes mellitus are at higher risk of infection. If any severe infection develops infliximab should be temporarily withdrawn and the need for antibiotics considered.

A recent report from the British Society of Rheumatology Biologics Register found that the overall risk of serious infections in rheumatoid arthritis patients was not increased by antiTNF alpha therapy compared to those on standard DMARD therapy. There were, however, more opportunistic infections, such as histoplasmosis, and more skin and soft tissue infections in the cohort on biologic therapy (Dixon et al 2007).

All TNF blockers carry a warning about reactivation of TB (black box for infliximab) (Centocor ${ }^{\circledR}$, Remicade $^{\circledR}$ data sheet). The boxed warning advises prescribing physicians to screen all patients for TB before initiation of therapy, to pretreat those with latent disease and to monitor patients for signs and symptoms of TB throughout the treatment course with infliximab. In a recent review of 130 cases of infliximab-associated tuberculosis reported to the FDA, many cases occurred in patients with a negative tuberculin skin test but with risk factors for TB. A fatal outcome was reported in 19 individuals (Raval et al 2007). Most cases occur shortly after initiation of treatment. Presentation may be with disseminated or military tuberculosis. A screening chest $\mathrm{x}$ ray is essential and testing for tuberculid sensitivity should be considered.

\section{Infusion reactions}

Another commonly reported event, occurring in around $20 \%$ of patients, is an infusion reaction especially during the first few infusions. This is usually defined as any adverse event occurring during or within 1 to 2 hours post infusion. The most common symptoms and signs are fever, chills, nausea and rarely chest pain, labile blood pressure, pruritus and urticaria. Slowing the rate of infusion and premedication with an antihistamine may ameliorate the reaction. Those who develop anti-infliximab antibodies seem particularly prone to reactions. Anaphylactic reactions have been reported (less than $1 \%$ of patients) in post-marketing surveillance. A guideline for the prevention and treatment of infusion reactions has recently been published (Lecluse et al 2008).

\section{Hepatotoxicity}

Asymptomatic elevation in transaminases (alanine aminotransferase more than aspartate aminotransferase) has been documented in clinical trials both when infliximab was used as a montherapy and in combination with other immunosuppressives. There is a tendency for the abnormalities to resolve or decrease with continued infliximab with consideration given to altering concomitant medication. Severe liver injury including reversible cholestasis and liver failure, occurring between a few weeks after commencement of infliximab to 1 year post initiation, has been reported rarely. Reactivation of hepatitis B has occurred in chronic carriers.

\section{Immunogenicity}

Anti-infliximab antibodies have been detected in $27 \%$ to $47 \%$ of infliximab-treated rheumatoid arthritis patients and appear to correlate with decreased serum infliximab levels and the need for escalating dosing regimens and possibly the loss of efficacy seen in some patients with time (Haraoui et al 2006; Wolbink et al 2006). The concomitant use of methotrexate may prevent development of these antibodies (Bendtzen et al 2006). This has not been investigated in phase 3 studies in psoriasis.

\section{Malignancy}

An increased risk of lymphoma has been noted in the clinical portion of trials of TNF blockers. It is not clear whether this risk relates to disease severity, previous treatments or a combination of factors. An increased risk of lymphoma is recognized in severe rheumatoid arthritis, psoriasis and systemic inflammation. Confounding factors include previous phototherapy, sun exposure, smoking and systemic therapies. A metaanalysis of 9 trials of infliximab or adalimumab in rheumatoid arthritis suggested a 3-fold increased risk of malignancy in the anti TNF alpha-treated group (OR 3.3, 95\% CI 1.2-9.1) (Bongartz et al 2006). Cutaneous malignancies, specifically 
non-melanoma skin cancers accounted for 9/35 cancers. This apparent excess risk occurred mainly after high dose infliximab ( $\geq 6 \mathrm{mg} / \mathrm{kg}$ ) which exceeds the dose usually used in rheumatoid arthritis and in psoriasis. The malignancy rate in the controlled arms was unexpectedly low, raising the possibility that the apparent increased risk in the TNF blocker group is simply a reflection of the low rate of malignancy in the control arm. The validity and conclusions from the study have been debated and questioned by clinicians. (Dixon et al 2006). It is likely that clarification of the relative importance of risk factors will come from the long term treatment registers of patients receiving biological therapies.

An increased incidence of hepatosplenic T cell lymphoma has been reported with infliximab. One hundred cases worldwide have been reported with 8 associated with infliximab used to treat pediatric inflammatory bowel disease. All patients were receiving concurrent immunosuppressant and/or prednisolone (Mackey et al 2007).

\section{Cardiac failure}

Initially TNF inhibitors were trialled as a treatment for congestive heart failure (CHF). Lack of efficacy halted investigation. New or young age at onset CHF and CHF in the absence of identifiable risk factors have all been reported in post-marketing reports of TNF inhibitors. Withdrawal of infliximab usually leads to improvement/resolution of symptoms (Kwon et al 2003).

\section{Demyelination}

TNF has been implicated in the pathogenesis of multiple sclerosis (Arnason et al 1999). A causal relationship between infliximab and demyelinating disease remains to be established but optic neuritis, transverse myelitis and multiple sclerosis (MS) have all been reported in patients being treated with TNF blockers (Mohan et al 2001). A prior history of MS or optic neuritis or these conditions in a first degree relative necessitates caution in the use of infliximab.

\section{Auto antibodies/lupus like syndromes}

The development of antinuclear antibodies and anti-DNA antibodies appears to be relatively common with infliximab treatment (Poulalhon et al 2007). The clinical significance of these remains to be defined. The development of antibodies does not preclude further infusions unless signs or symptoms of lupus develop. Resolution of lupus-like syndromes usually occurs on cessation of infliximab.

\section{Other adverse events}

Post-marketing surveillance across specialities has revealed occasional reports of neutropenia, interstitial pneumonitis, thrombocytopenic purpura (idiopathic and thrombotic), vasculitis, erythema multiforme, toxic epidermal necrolysis, transverse myelitis, Guillain Barré syndrome and neuropathies. Weight gain has been reported in patients with inflammatory bowel disease and spondyloarthropathies treated with TNF antagonists. Similar findings in the psoriasis population (Gisondi et al 2007) are important as chronic plaque psoriasis has been shown to be associated with obesity and may even be a risk factor for the disease (Mallbris et al 2005; Naldi et al 2005).

\section{Comparative efficacy}

To date, there are no published trials directly comparing the efficacy of infliximab with systemic therapies such as methotrexate, ciclosporin or retinoids, or with other biological therapies. An indirect comparison of infliximab and other biological therapies was undertaken by NICE using methodology from the health technology assessment of etanercept and infliximab. This showed a greater efficacy in terms of PASI 75 with infliximab compared to efalizumab or etanercept (Scottish Medicines Consortium 2007). Further support was lent by the British Association of Dermatologists guideline for the management of psoriasis which, at the time of writing, noted that data from short-term individual studies suggested that infliximab may be the most efficacious biological therapy for psoriasis (Smith et al 2005).

\section{Combination therapy}

The synergistic effect of combining methotrexate and infliximab has been described in the rheumatology literature (Maini et al 1998). This may, in part, be the result of methotrexate-induced alteration in infliximab excretion. In Crohn's disease and rheumatoid arthritis, a lack of sustained response to infliximab, despite dose escalation, may prompt the addition of methotrexate which has been shown to decrease the development of human antichemeric antibodies (Maini et al 1998; Farrell et al 2001).

\section{The place of infliximab in clinical practice}

The National Institute for Health and Clinical Excellence (NICE) and the Scottish Medicines Consortium (SMC) have issued guidance about the place of infliximab in the treatment of adults with psoriasis and psoriatic arthritis. NICE is an 
independent organization responsible for providing national guidance on treatments and care for people using the NHS in England and Wales. Similarly, the SMC provides advice on newly licensed medicines in Scotland.

NICE recommends infliximab for patients with severe disease (PASI 20 or more and a DLQI of greater than 18) who have failed, are intolerant of or have contraindication to treatment with standard systemic therapies such as ciclosporin, methotrexate or PUVA. Continuation of infliximab beyond 10 weeks requires a PASI 75 response or a PASI 50 in addition to a 5-point reduction in the DLQI.

For adults with psoriatic arthritis, infliximab may be prescribed if there are 3 or more tender and 3 or more swollen joints in addition to treatment failure with at least 2 standard disease-modifying anti-rheumatic drugs administered individually or in combination. Infliximab is recommended only after intolerance of, or contraindications to, etanercept. An inability to self administer etanercept is considered an acceptable reason to progress to therapy with infliximab. For treatment to continue beyond 12 weeks an improvement in at least two of the four PsARC criteria (one of which has to be joint tenderness or swelling, with no worsening in any of the four criteria) is required.

The financial savings and costs associated with implementing the NICE guidelines on infliximab for psoriasis can be estimated, in the UK, using a template available at www.nice.org.uk/TA134.

In our own practice, infliximab is chosen for individuals with severe disease, frequently involving skin and joints, in whom rapid control is desirable. Delivery of the drug is by intravenous infusion in a hospital setting permitting us to monitor concordance with treatment, efficacy and to readily assess side effects. Intravenous delivery is particularly suitable for those with severe joint disease who may not be dextrous enough to self administer treatment.

\section{Conclusion}

Studies have demonstrated that infliximab is an effective treatment for chronic plaque psoriasis with $90 \%$ of patients becoming clear or having minimal disease activity after $5 \mathrm{mg} / \mathrm{kg}$ infused at weeks 0,2 and 6 . Thereafter, maintenance infusions at approximately 8-weekly intervals may encourage prolonged efficacy. There are fewer data about use in erythrodermic psoriasis and in generalized pustular psoriasis but the drug appears to be effective in this setting. In some patients with very severe or unstable disease concomitant systemic therapy may give added benefit. Treatment with infliximab is continued beyond 10 weeks only when an objective response (measured using PASI and DLQI scores) can be demonstrated.

Psoriasis tends to be a chronic disease and those who are most severely affected frequently require medical intervention over many years. Treatment regimens need to be tailored to individual patients taking in to consideration co-morbidities, the pattern, extent and psychological impact of psoriasis. A multi-disciplinary approach is often helpful. The establishment of registries such as the BAD biologics register (BADBIR) will undoubtedly yield useful and important data on long term safety and efficacy of biological therapies such as infliximab.

\section{Disclosures}

JAL has undertaken trials for Schering Plough, Merck Serono and Abbott. ADB has consulted to, undertaken trials for and lectured on behalf of Schering Plough, Wyeth, Merck Serono, Abbott and Janssen Cilag.

\section{References}

Antoni C, Kavanaugh A, Kirkham B, et al. 2005a. Sustained benefits of infliximab therapy for dermatologic and articular manifestations of psoriatic arthritis: results from the Infliximab Multinational Psoriatic Arthritis Controlled Trial. Arthritis Rheum, 52:1227-36.

Antoni C, Krueger GG, de Vlam K, et al. 2005b. Infliximab improves signs and symptoms of psoriatic arthritis: results of the IMPACT 2 trial. Ann Rheum Dis, 64:1150-75.

Arnason BGW 1999. The lenercept Multiple Sclerosis Study Group and the University of British Columbia MS/MRI Analysis Group. TNF neutralisation in MS: results of a randomised. placebo controlled multicentre study. Neurology, 53:457-65.

Bendtzen K, Geborek P, Svenson M et al. 2006. Individualized monitoring of drug bioavailability and immogenicity in rheumatoid arthritis patients treated with tumour necrosis factor alpha inhibitor infliximab. Arthritis Rhem, 54:3782-89.

Benoit S, Toksoy A, Brocker EB et al. 2004. Treatment of recalcitrant pustular psoriasis with infliximab: effective reduction of chemokine expression. Br J Dermatol, 150:1009-12.

Bianchi L, Bergamin A, de Felice C, et al. 2005. Remission and time of resolution of nail psoriasis during infliximab therapy. $J$ Am Acad Dermatol, 52:736-7.

Bongartz T, Sutton AJ, Sweeting MJ, et al. 2006. Anti-TNF antibody therapy in rheumatoid arthritis and the risk of serious infections and malignancies: systematic review and meta-analysis of rare harmful effects in randomized controlled trials. JAMA, 295:2275-85.

Bundu-Kamara S. 2002. Therapeutic Management of psoriasis Hospital Pharmacist 9:191-99.

Burd RM. 2006. Psoriasis: a general overview. Br J Hosp Med, 67:259-62.

Camp RDR, El-Rachkidy RG, Young HS, et al. 2006. Immunological evidence for increasing reactivity to secreted Streptococcus pyogenes proteins in chronic plaque psoriasis: FC-13. Br J Dermatol, 154(Suppl 1):5.

Capon F, Di Meglio P, Szaub J, et al. 2007. Sequence variants in the genes for the interleukin-23 receptor (IL23R) and its ligand (IL12B) confer protection against psoriasis. Hum Genet, 122(2):201-6.

Carlin CS, Feldman SR, Krueger JG, et al. 2004. A 50\% reduction in the Psoriasis Area and Severity Index (PASI 50) is a clinically significant endpoint in the assessment of psoriasis. J Am Acad Dermatol 50:859-66. 
Cather J, Menter A . 2005. Combining traditional agents and biologics for treatment of psoriasis. Semin Cut Med Surg, 24:37-45.

Centocor Inc. 2006. Remicade (infliximab) package insert: Chicago, Il, USA available at www.gene.com/gene/products/information/pdf/ raptivaprescribing.pdf.

Clements SE, Abdul-Ghaffar S, Griffiths CE. 2006. Biological therapy for psoriasis. Br J Hosp Med(Lond), 67:184-7.

Collamer A, Guerrero KT, Henning JS, et al. 2008. Psoriatic skin lesions induced by tumour necrosis factor antagonist therapy: a literature review and potential mechanisms of action .Arthritis Care and Research, 57:996-1001.

Cookson WO. Ubhi B. Lawrence R et al. 2001. Genetic linkage of childhood atopic dermatitis to psoriasis susceptibility loci. Nature Genetics, 27(4):372-3.

Dixon W, Silman A. 2006. Is there an association between anti-TNF antibody therapy in rheumatoid arthritis and risk of malignancy and serious infection? Commentary on the meta analysis by Bongartz et al. Arthritis Res Ther, 8:11.

Dixon WG, Symmons DP, Lunt M, et al. 2007.Rates of serious infection, including site-specific and bacterial intracellular infection, in rheumatoid arthritis patients receiving anti-tumor necrosis factor therapy: results from the British Society for Rheumatology Biologics Register (BSRBR). Arthritis Rheum, 56:2896-904.

Elder JT. 2006.PSORS 1: linking genetics and immunology. $J$ Invest Dermatol, 126:1205-6.

Espinoza LR, Cuellar ML, Silveira JH. 1992. Psoriatic arthritis. Curr Opin Rheumatol, 4:470-8.

Farrel RJ, Alsahli M, Falchuk KR, et al. 2001. Human ant-chimaeric antibody levels correlate with a lack of response and infusion reactions following infliximab therapy. Gastroenterology, 120(Suppl 1):A69 abstract 364 .

Feldman SR, Krueger GG. 2005. Psoriasis assessment tools in clinical practice. Ann Rheum Dis, 64(Suppl ii):65-8.

Feldman SR, Gordon KB, Bala M, et al. 2005. Infliximab results in significant improvement in the quality of life of patients with severe psoriasis: a double - blind placebo-controlled trial. J Am Acad Dermatol, $152: 954-60$

Finlay AY. 2005. Current severe psoriasis and the rule of tens. Br J Dermatol, 152:861-7.

Gelfland JM, Weinstein R, Porter SB, et al. 2005. Prevalence and treatment of psoriasis in the United Kingdom: a population - base study. Arch Dermatol, 141:1537-41.

Gelfand JM, Neimann AL, Shin DB, et al. 2006. Risk of myocardial infarction in patients with psoriasis. JAMA, 296:1735-41.

Gilhar A, David M, Ullman T, et al. 1997. T-lymphocyte dependence of psoriatic pathology in human psoriatic skin grafted to SCID mice. J Invest Dermatol, 109:283-8.

Gisondi P, Cotena C, tessari G, et al. 2007. Anti-tumour necrosis factor alpha therapy increases bodt weight in patients with chronic plaque psoriasis: a retrospective cohort study. J Eur Acad Dermatol, 22:341-4.

Gladman D, Antoni C, Mease P, et al. 2005. Psoriatic arthritis: epidemiology, clinical features, course and outcome. Ann Rheum Dis, 64(SuppI 1):14-7.

Gottlieb AB, Evans R, Li S, et al. 2004. Infliximab induction therapy for patients with severe plaque-type psoriasis: a randomised double-blind, placebo-controlled trial. J Am Acad Dermatol, 51:534-42.

Gudmundsdottir AS, Sigmundsdotir H, Sigurgeirsson B, et al. 1999. Is an epitope on keratin 17 a major target for autoreactive $\mathrm{T}$ lymphocytes in psoriasis? Clin Exp Dermatol. 117:580-6.

Haraoui B, Cameron L, Ouellet M, et al. 2006. Anti-infliximab antibodies in patients with rheumatoid arthritis who require higher doses of infliximab to achieve or maintain a clinical response. J Rheumatol, 33:31-6.

Hochberg MC, Lebwohl MG, Plevy, et al. 2005. The benefit/risk profile of TNF - blocking agents: findings of a concensus panel. Semin Arthritis Rheum, 34:819-36.

Hyrich KL, Symmons DPM, Watson KD, et al. 2006. Pregnancy outcome in women who were exposed to anti-tumour necrosis factor agents: results from a national population register. Arthritis Rheum, 54:2701-8.
Kavanaugh A, Krueger GG, Beutler A, et al. 2007. Infliximab maintains a higher degree of clinical response through 1 year of treatment: results from the IMPACT 2 trial. Ann Rheum Dis, 66:498-505.

Krueger JG. 2002. The immunological basis for the treatment of psoriasis with new biological agents. $J$ Am Acad Dermatol, 46:1-23.

Kwon HJ, Cote TR, Cuffe MS, et al. 2003. Case reports of heart failure after therapy with tumour necrosis factor antagonist. Ann Intern Med, 138:807-11.

Lebowohl M. 2003. Psoriasis. Lancet, 361:1197-204.

Lebowohl M, Callen JP. 2006. Obesity, smoking and psoriasis. JAMA, 295:208-10.

Lecluse LLA, Piskin G, Mekkes JR, et al. 2008. Review and expert opinion on the prevention and treatment of infliximab-related infusion reactions. Br J Dermatol, 159:527-36.

Lewis V, Finlay AY. 2004. 10 years experience of the Dermatology Life Quality index (DLQI) J Invest Dermatol Symp Proc, 9:169-8.

Lewis TG, Tuchinda C, Lim HW, et al. 2006. Life threatening pustular and eythrodermic psoriasis responding to infliximab. J Drugs Dermatol, 5:546-8.

Lomholt G. 1963. Psoriasis: prevalence, spontaneous course, and genetics. GEC, GAD, Copenhagen.

Mackkey AC, Green L, Liang LC, et al. 2007. Hepatosplenic T cell lymphoma associated with infliximab use in young patients treated for inflammatory bowel disease. J Pediatr Gastroenterol Nutr, 44:265-7.

Maini RN, Breedveld FC, Kalden JR, et al. 1998. Therapeutic efficacy of multiple intravenous infusions of anti-tumour necrosis factor alpha monoclonal antibody combined with low dose weekly methotrexate in rheumatoid arthritis. Arthritis Rheum, 41:1552-63.

Mallbris L, Arke O, Granath F, et al. 2004. Increased risk for cardiovascular mortality in psoriasis inpatients but not outpatients. Eur J Epidemiol, 19:225-30.

Mallbris L, Larsson P, Bergqvist S, et al. 2005. Psoriasis phenotype at disease onset: clinical characterisation of 400 adult cases. $J$ Invest Dermatol, 124:499-504.

Martínez-Morán C, Sanz-Muñoz C, Morales-Callaghan AM, et al. 2007. Pustular psoriasis induced by infliximab. JEur Acad Dermatol Venereol, $21: 1424-6$

Mease P, Goffe B. 2005. Diagnosis and treatment of psoriatic arthritis. $J$ Am Acad Dermatol, 52:1-19.

Mease PJ, Antoni CE, Gladman DD et al. 2005. Psoriatic arthritis assessment tools in clinical trials. Ann Rheum Dis, 64:ii49-ii54.

Mease PJ, Menter A. 2006. Quality of life issues in psoriasis and psoriatic arthritis:Outcome measure and therapies from a dermatological perspective. J Am Acad Dermatol, 50:859-66.

Menter M, Alan MD, Cush JM, et al. 2004. Successful treatment of pediatric psoriasis with infliximab. Paed Dermatol, 21:87-8.

Menter A, Feldman SR, Weinstein GD, et al. 2007. A randomised comparison of continuous vs intermittent infliximab maintenance regimens over 1 year in the treatment of moderate-severe plaque psoriasis. J Am Acad Dermatol, 56:31.e1-15.

Mohan N, Edwards ET, Cupps TR, et al. 2001. Demyelination occurring during anti-tumour necrosis factor alpha for inflammatory arthritidies. Arthritis Rheum, 44:2862-9.

Moll JM, Wright V. 1973. Psoriatic arthritis. Semin Arthritis Rheum 3:55-78.

Mossner R, Thaci D, Mohr J, et al. 2008. Manifestation of palmoplantar pustulosis during or after infliximab therapy for plaque type psoriasis: report on five cases. Arch Dermatol Res, 30:101-5.

Naldi L, Chatenoud L, Linder D, et al. 2005. Cigarette smoking, body mass index, and stressful life events as risk factors for psoriasis: results from an Italian case control study. $J$ Inves Dermatol, 125:61-7.

NICE. 2006. Infliximab for the treatment of adults with psoriasis- Final Scope. NICE single technology appraisal [online]. URL: www.nice. org.uk/TA134.

Nickoloff BJ. 1999. The cytokine network in psoriasis. Arch Dermatol, 127:871-84. 
Pereira TM, Vieira AP, Fernandes JC, et al. 2006. Anti-TNF alpha in childhood pustular psoriasis. Dermatology, 213:350-2.

Poikolainen K, Karvonen J, Pukkala E. 1999. Excess mortality related to alcohol and smoking among hospital treated patients with psoriasis. Arch Dermatol, 135:1490-3.

Poulalhon N, Begon E Lebbe C, et al. 2007. A follow up study in 28 patients treated with infliximab for severe recalcitrant psoriasis: evidence for efficacy and high incidence of biological immunity. Br J Dermatol, 156;329-36.

Rapp SR, Feldman SR, Exum ML, et al. 1999. Psoriasis causes as much disability as other major medical diseases. $J$ Am Acad Dermatol, 41:401-7.

Raval A, Akhavan-Toyserkani G, Brinker A et al. 2007. Brief communication: characteristics of spontaneous cases of tuberculosis associated with Infliximab. Ann Int Med, 147;699-702.

Reich K, Nestle FO, Papp K, et al. 2005. Infliximab induction and maintenance for moderate-to-severe psoriasis: a phase III, multi centre, double-blind trial. Lancet, 366:1367-74.

Rich P, Griffiths CE, Reich K, et al. 2008. Baseline nail disease in patients with moderate to severe psoriasis and response to treatment with infliximab during 1 year. J Am Acad Dermatol, 58:224-31.

Rigopoulos D, Gregoriou S, Stratigos A, et al. 2008. Evaluation of the efficacy and safety of infliximab on psoriatic nails: an unblinded, nonrandomized, open-label study. Br J Dermatol, 159:453-6.

Rott S, Kuster RM, Mrowietz U. 2007. Successful treatment of severe psoriatic arthritis with infliximab in an 11 year old child suffering from linear psoriasis along the lines of Blaschko. Br J Dermatol, 157:191-2.

Routhouska SB, Sheth PB, Korman NJ. 2008. Long-term management of generalized ustular psoriasis with infliximab: case series. J Cut Med Surg, 12:184-8.

Scheinfeld N. 2004. A comprehensive review and evaluation of the side effects of tumour necrosis factor alpha blockers, etanercept, infliximab and adalimumab. Dermatol Treat, 15:280-94.

Scher RK. 1985. Psoriasis of the nail. Dermatol Clin, 3:387-94.

Schmick K, Grabbe J. 2004. Recalcitrant, generalized pustular psoriasis: rapid and lasting therapeutic response to anti tumour necrosis factor-alpha antibody (infliximab). Br J Dermatol, 150:367.
Scottish Medicines Consortium guidance on infliximab $100 \mathrm{mg}$ powder for intravenous infusion (Remicade) No (318/06) 2007.

Setty AR, Curhan G, Choi HK. 2007. Obesity, waist circumference, weight change and the risk of psoriasis in women. Arch Intern Med, $167: 1670-5$

Smith CH, Anstey AV, Barker, et al. 2005. British Association of Dermatologists guideline for the use of biological interventions in psoriasis. Br J Dermatol, 153:486-97.

Smith CH, Barker JN. 2006. Psoriasis and its management. BMJ, 333:380-4.

Takahashi MD, Castro LG, Romiti R. 2007. Infliximab, as sole or combined therapy, induces rapid clearing of erythrodermic psoriasis. Br J Dermatol, 157:828-31.

Telfer NR, Chalmers RJ, Whale K, et al. 1992. The role of streptococcal infection in the initiation of guttate psoriasis. Arch Dermatol, 128:39-42.

Trent JT, Kerdel FA. 2004. Successful treatment of Von Zumbush pustular psoriasis with infliximab. J Cut Med Surg, 8:224-8.

Uyanik BS, Ari Z Omur E, et al. Serum lipids and apolipoproteins in patients with psoriasis. 2002. Clin Chem Lab Med, 40:65-8.

Valdimarsson H, Sigmundsdottir H, Jonsdottir I. 1997. Is psoriasis induced by streptococcal superantigens and maintained by M-protein-specific T cells that cross react with keratin? Clin Exp Dermatol, 107(Supp 1):21-4.

Van de Kerkhof P. 2003. Biologic agents offer hope for psoriasis. Business briefing: European Pharmacotherapy. p124-8.

Vieira Serrao V, Martins A, Lopes MJ .2008. Infliximab in recalcitrant generalised pustular arthropathic psoriasis. Eur J Dermatol, 18:71-2.

Weisenseel P, Prinz JC. 2006. Sequential use of infliximab and etanercept in generalized pustular psoriasis Cutis, 78:197-9.

Wolbink GJ, Vis M, Lems W, et al. 2006. Development of anti-infliximab antibodies and relationship to clinical response in patients with rheumatoid arthritis. Arthritis Rheum, 54:711-5.

Wollina U, Hansel G, Koch A, et al. 2008. Tumour necrosis factor alpha inhibitor induced psoriasis or psoriasiform exanthemata: first 120 cases from the literature including a series of six new patients. Am J Clin Dermatol, 9:1-14 
